\title{
Delayed Sciatic Nerve Injury Resulting From Myositis Ossificans Traumatica
}

\author{
Zhe Guan, MS, Thomas J. Wilson, MD, Jon A. Jacobson, MD, \\ Todd C. Hollon, MD, Lynda J.-S. Yang, MD, PhD
}

\begin{abstract}
A motorcyclist sustained multiple-system trauma, including a left buttock hematoma requiring decompression and evacuation. Presentation for severe hip pain and lower extremity weakness was delayed. Imaging revealed myositis ossificans traumatica compressing the sciatic nerve in the buttock. The patient underwent sciatic nerve decompression with resection of heterotopic calcification, resulting in improvement in pain and left lower extremity function. This case illustrates the contrast in differential diagnosis of peripheral nerve injury immediately posttrauma and that occurring in a slow, delayed fashion posttrauma. Myositis ossificans may be an underrecognized complication of trauma but should be considered in cases of delayed peripheral nerve injury after trauma.
\end{abstract}

\section{Introduction}

Neurological deficits after peripheral nerve injury may occur either immediately due to direct injury at the site of impact or in a delayed fashion due to a variety of processes that cause secondary insult, including hematoma formation, delayed ischemia, or iatrogenic injury. Pelvic trauma/fractures are associated with an increased risk of nerve injury, particularly to the sciatic nerve [1]. When trauma is associated with hematoma formation, myositis ossificans traumatica can occur in the subsequent months. In the following case report, we recount the case of a 43-year-old motorcycle trauma victim who developed delayed, progressive, complete paralysis of the lower leg with intractable pain due to sciatic nerve compression from myositis ossificans traumatica.

\section{Case Presentation}

A 43-year-old man was injured in a motorcycle accident and sustained multiple-system trauma. His injuries included sacral fracture, right femoral neck fracture, lumbar vertebral fracture, and ruptured bladder. He underwent emergent femoral neck fixation and bladder repair. His sacral and vertebral fractures were treated nonoperatively. During his hospital course, he was suspected to have left gluteal compartment syndrome, a rare disorder affecting 1 or more of the 3 anatomic gluteal compartments: the gluteus maximus compartment, the gluteus medius and minimus compartment, and the tensor fasciae latae compartment. The disorder typically presents with swelling, redness, and tenderness over the buttock, ipsilateral hip pain, and sciatic nerve deficits. The patient underwent emergent fasciotomy followed by vacuum-assisted wound closure. He subsequently developed signs of blood loss and was taken back to the operating room, where he was found to have a large left buttock hematoma and iatrogenic ruptured left superior gluteal artery. Postoperatively, he had full strength in his lower extremities. He was referred for a 3-week course of inpatient rehabilitation due to his fractures and hospital-associated deconditioning, after which he was discharged home with continued outpatient physical therapy. Four weeks after discharge, he complained of new onset of left foot drop and severe left hip and buttock pain radiating to the left leg, which worsened with movement and weight bearing. The symptoms were attributed to nerve injury sustained during surgery, although the patient reported 
normal left lower extremity function 7 weeks after hematoma evacuation.

At our initial neurosurgical evaluation, which occurred 40 weeks after his last surgery, the patient was unable to be seated due to severe left hip pain and instead lay on his right side on the examining room table. Manual muscle testing was significant for Medical Research Council 0/5 strength in dorsiflexion, plantar flexion, eversion, and inversion of the left ankle, 4/5 flexion and extension of the left knee, $3 / 5$ in hip flexion, $4 / 5$ strength hip extension, and hypoesthesia in the left sural, superficial peroneal, and deep peroneal nerve distributions. The patient reported significant pain in the left buttock, and his examination was confounded by pain inhibition; the most significant weakness was in hamstring and lower leg function.

Electromyography was consistent with a severe left sciatic neuropathy without any evidence of axonal continuity to sampled muscles innervated by sciatic nerve (Tables 1 and 2). Normal electromyography of the gluteus maximus and vastus medialis was consistent with localization of the lesion to the buttock; because pain inhibition was the likely cause of weakness in hip flexion, the iliopsoas was not thought to be denervated and therefore was not targeted in the electrodiagnostic examination. Magnetic resonance imaging (MRI) was performed (Figure 1), which showed marked enlargement of the left sciatic nerve extending from the pelvic exit to the level of the lesser trochanter, compression of the sciatic nerve from probable heterotopic ossification, as well as muscle edema in the left hip and thigh muscles. With respect to imaging, heterotopic ossification and myositis ossificans can be difficult to identify by $M R I$, especially in the early stages, when there is significant soft tissue edema [2]. Computed tomography (CT) is an ideal imaging method to accurately demonstrate the presence and extent of soft tissue calcification and ossification [2]. Therefore, the patient also underwent pelvic (buttock) CT (Figure 2) to confirm the presence of heterotopic ossification and for preoperative consideration. Extensive heterotopic ossification was found in the left posterior gluteal tissues with compression of the left sciatic nerve at the sciatic notch. Myositis ossificans traumatica was suspected based on history and imaging findings.
The patient underwent left sciatic nerve exploration via a posterior approach. The gluteus maximus was split and the greater sciatic notch located. A large sheet of bone was impinging on the sciatic nerve. The heterotopic bone was removed piecemeal and later identified as mature bone on pathologic analysis.

The patient reported relief of left hip, buttock, and leg pain after surgery, and he regained the ability to sit comfortably for extended periods, significantly improving his quality of life. He was pleased to be able to attend the wedding of a close family member and to remain seated for the full duration of the ceremony without pain. At the 6-week follow-up visit, he remained pain-free. He also regained full strength in knee flexion and knee extension, although more distal function remained $0 / 5$ except for twitch in dorsiflexion.

\section{Discussion}

Myositis ossificans traumatica is the heterotopic ossification of muscle after trauma. Although nerve injury secondary to myositis ossificans traumatica is a rare event, this case serves to illustrate the importance of evaluating and recognizing delayed neurologic deficits after trauma. Peripheral nerve injury has been reported to occur in $1 \%-2 \%$ of all trauma cases, with the majority occurring as a result of direct nerve injury [1]. Pelvic fractures appear to have the greatest risk of nerve injury out of all traumatic injuries, and usually affect the sciatic nerve, lumbosacral plexus, or femoral nerve [1].

Delayed neurologic deficits after trauma require expeditious evaluation because a variety of causes can potentially be addressed with intervention. The differential diagnosis for subacute neurologic injury includes compression from hematoma enlargement, localized edema, compartment syndrome, arterial or venous aneurysms/pseudoaneurysms, infection/abscess, iatrogenic injury (eg, bracing, casting, compression from operative hardware, nerve strangulation from a suture), and myositis ossificans traumatica.

In our patient, sciatic nerve injury occurred secondary to myositis ossificans, which has only rarely been reported with various underlying conditions [3-6]. Myositis ossificans can be a relatively common

Table 1

Lower extremity electromyography

\begin{tabular}{|c|c|c|c|c|c|c|c|c|c|c|}
\hline \multirow[b]{2}{*}{ Muscle } & \multicolumn{5}{|c|}{ Insertional/Spontaneous } & \multicolumn{5}{|c|}{ Voluntary Motor Unit Potential } \\
\hline & Insertion & Other & P-wave & $\begin{array}{l}\text { Fibrillation } \\
\text { Potentials }\end{array}$ & Fasciculations & Effort & Recruitment & Amplitude & Duration & Polyphasia \\
\hline Vastus medialis & Normal & 0 & 0 & 0 & 0 & $\mathrm{~N}$ & $\mathrm{~N}$ & $\mathrm{~N}$ & $\mathrm{~N}$ & $\mathrm{~N}$ \\
\hline Anterior tibialis & Increasing & 0 & Sustained & $3+$ & 0 & $\mathrm{~N}$ & 0 & - & - & - \\
\hline Medial gastrocnemius & Increasing & 0 & Sustained & $3+$ & 0 & $\mathrm{~N}$ & 0 & - & - & - \\
\hline Internal hamstring & Increasing & 0 & Sustained & $3+$ & 0 & $\mathrm{~N}$ & 0 & - & - & - \\
\hline Biceps femoris, short & Increasing & 0 & Sustained & $2+$ & 0 & $\mathrm{~N}$ & 0 & - & - & - \\
\hline Gluteus maximus & Normal & 0 & 0 & 0 & 0 & $\mathrm{~N}$ & $\mathrm{~N}$ & $\mathrm{~N}$ & $N$ & $2+$ \\
\hline
\end{tabular}


Table 2

Lower extremity nerve conduction studies

\begin{tabular}{|c|c|c|c|c|c|c|c|}
\hline \multirow[b]{2}{*}{ From } & \multirow[b]{2}{*}{ To } & \multicolumn{2}{|c|}{ Amplitude (mV or $\mu \mathrm{V})$} & \multicolumn{2}{|c|}{ Latency (ms) } & \multicolumn{2}{|c|}{ Velocity $(\mathrm{m} / \mathrm{s})$} \\
\hline & & Right & Left & Right & Left & Right & Left \\
\hline Calf & Ankle & 8.6 & No response & 3.6 & - & 48.3 & - \\
\hline Ankle & Extensor digitorum brevis & 3.1 & No response & 5.1 & - & - & - \\
\hline Below knee & Extensor digitorum brevis & 2.7 & No response & 11.8 & - & 43.9 & - \\
\hline Below knee & Anterior tibialis & - & No response & - & - & - & - \\
\hline Ankle & Abductor hallucis & - & No response & - & - & - & - \\
\hline
\end{tabular}

consequence of trauma and has been reported to occur in up to $53 \%$ of spinal cord injury patients [7]. Exact rates are difficult to decipher because of differences in inclusion criteria and variation in follow-up periods. In addition, many patients who develop myositis ossificans are asymptomatic, and screening of asymptomatic subjects varies from study to study. The pathogenesis of myositis ossificans is not well understood, but studies have shown that expression of bone morphogenic protein 2 plays a key role by inducing neuroinflammation and attracting osteogenic stem cells to the nerve [8,9]. Currently, the only treatment for neuropathy associated with myositis ossificans is surgical decompression.

Recurrence of ossification is a significant complication, but the timing of surgery can vary depending on the clinical situation. Although the patient may have pain, surgical resection is usually delayed for 1 year until the heterotopic bone is considered to be mature and no further ossification is expected. However, some evidence is accumulating to support early intervention without a significant difference in recurrence [10]. If there are significant negative consequences of delayed surgery (eg, potential joint contracture) [10], or in this case, a progressive motor deficit, consideration of earlier intervention is warranted, as conservative management (eg, pain management, physical therapy) did not improve the patient's symptoms. It should be noted that myositis ossificans is difficult to treat, as it can be a chronic spreading process or it may spontaneously resolve. Maturity of the heterotopic bone can be difficult to assess with preoperative imaging; however, in our patient, the pathological diagnosis of mature bone was reassuring that the timing of our earlier surgical intervention was appropriate. In the case of nerve compression/progressive neurological deficit after a traumatic injury in the subacute period, clinical suspicion should lead to timely evaluation to identify the site and etiology of the new neurological deficit. Since a focal area of compression of the sciatic nerve was identified that corroborated the clinical examination, our patient underwent earlier surgical intervention. Compared to central nervous system lesions, peripheral nerve lesions are more likely to present with weakness and atrophy of individual muscles, sharply demarcated areas of sensory symptoms, and autonomic symptoms. Preserving and potentially regaining the affected peripheral nerve's function depends significantly on appropriate timing of diagnosis and decompression.

This case presentation highlights the importance of proper diagnosis of peripheral nerve injury that occurs in a delayed fashion after trauma. The error in this case occurred when a delayed deficit was attributed to the initial trauma and not further investigated. Because even slight delays in evaluation may result in permanent disability, clinicians should maintain a high index of suspicion for secondary nerve injury and order
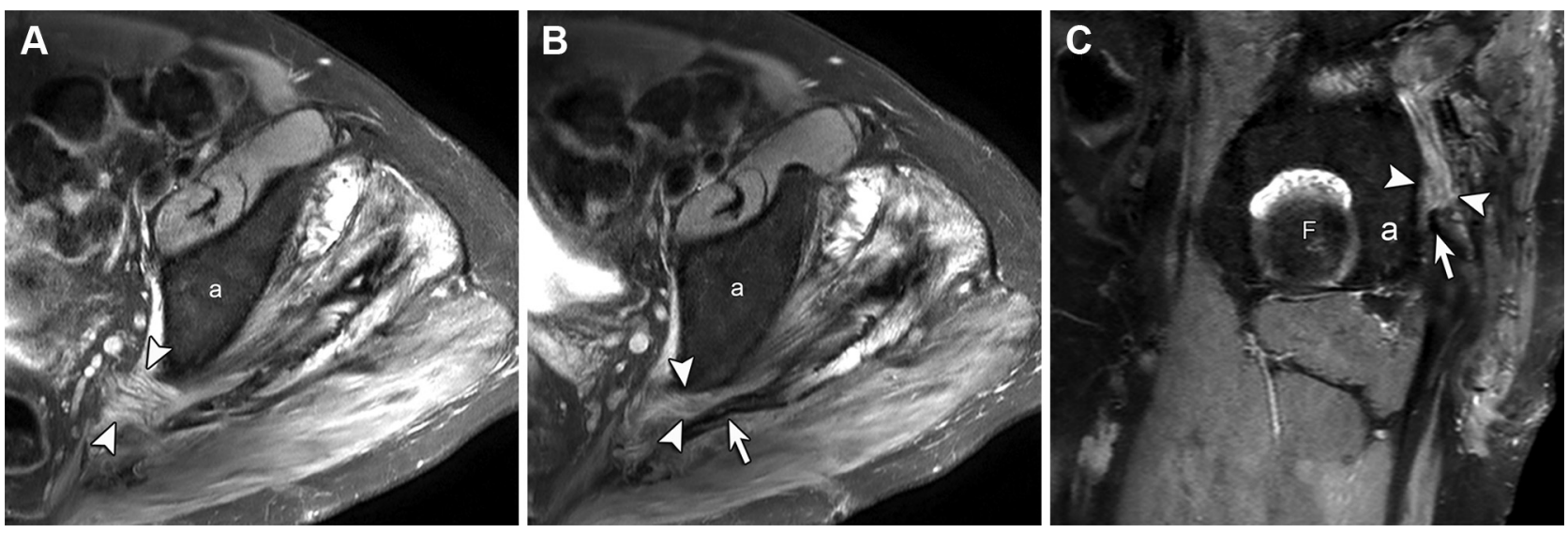

Figure 1. Sequential axial proton-density magnetic resonance images of left hip with fat saturation show (A) enlargement and edema of the sciatic nerve (arrowheads; $\mathrm{a}$ = acetabulum) with (B) more distal compression (arrowheads) between the heterotopic ossification (arrow) and acetabulum (a). Similar findings are shown in the sagittal plane $(C)$. Note high-signal edema in adjacent gluteal muscles $(F=$ femoral head; $a=$ acetabulum). 


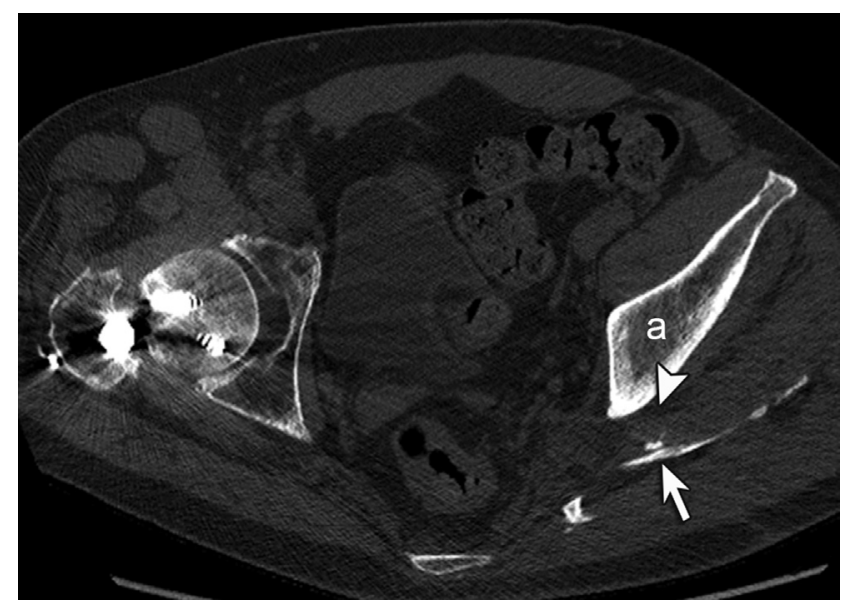

Figure 2. Noncontrast computed tomographic image of the pelvis shows compression of sciatic nerve (arrowhead) between acetabulum (a) and heterotopic ossification (arrow). Note internal fixation of proximal right femur fracture.

appropriate imaging studies when a compatible history is obtained. In our case, the patient's intervention was severely delayed, causing significant disability associated with a decreased quality of life.

\section{Conclusion}

This case presentation illustrates the contrast in the differential diagnosis of peripheral nerve injury immediately after trauma and that which occurs in a slow delayed fashion after trauma. Investigating the origin of delayed symptoms is important, as intervention is feasible for a number of causes. Myositis ossificans may be an underrecognized complication of trauma, and although rare, should be considered in the differential diagnosis of delayed peripheral nerve injury after trauma. This condition, in which heterotopic calcification of muscle occurs after traumatic hematoma formation and resolution, can progressively compromise the sciatic nerve several months after the initial injury. Astute diagnosis in the context of complex trauma and surgical decompression of the sciatic nerve are critically important to prevent or limit development of permanent neurologic deficits.

\section{References}

1. Taylor CA, Braza D, Rice JB, Dillingham T. The incidence of peripheral nerve injury in extremity trauma. Am J Phys Med Rehabil 2008; $87: 381-385$.

2. Tyler P, Saifuddin A. The imaging of myositis ossificans. Semin Musculoskelet Radiol 2010;14:201-216.

3. Beauchesne RP, Schutzer SF. Myositis ossificans of the piriformis muscle: An unusual cause of piriformis syndrome. A case report. J Bone Joint Surg Am 1997; 79:906-910.

4. Goddyn C, Passuti N, Leconte R, Redon H, Gouin F. Sciatic nerve compression related to ossification of the sacrospinous ligament secondary to pelvic balance abnomalities. Orthop Traumatol Surg Res 2009;95:645-648.

5. Kleiman SG, Stevens J, Kolb L, Pankovich A. Late sciatic-nerve palsy following posterior fracture-dislocation of the hip. A case report. J Bone Joint Surg Am 1971;53:781-782.

6. Reinstein L, Eckholdt JW. Sciatic nerve compression by preexisting heterotopic ossification during general anesthesia in the dorsal lithotomy position. Arch Phys Med Rehabil 1983;64: 65-68.

7. Colachis SC 3rd, Clinchot DM, Venesy D. Neurovascular complications of heterotopic ossification following spinal cord injury. Paraplegia 1993;31:51-57.

8. McCarthy EF, Sundaram M. Heterotopic ossification: A review. Skeletal Radiol 2005;34:609-619.

9. Salisbury E, Rodenberg E, Sonnet C, et al. Sensory nerve induced inflammation contributes to heterotopic ossification. J Cell Biochem 2011;112:2748-2758.

10. Chen S, Yu SY, Yan H, et al. The time point in surgical excision of heterotopic ossification of post-traumatic stiff elbow: recommendation for early excision followed by early exercise. J Shoulder Elbow Surg 2015;24:1165-1171.

\section{Disclosure}

Z.G. University of Michigan Medical School, University of Michigan, Ann Arbor, MI Disclosure: nothing to disclose

T.J.W. Department of Neurosurgery, University of Michigan, Ann Arbor, MI Disclosure: nothing to disclose

J.A.J. Department of Radiology, University of Michigan, Ann Arbor, Ml Disclosure: nothing to disclose
T.C.H. Department of Neurosurgery, University of Michigan, Ann Arbor, Ml Disclosure: nothing to disclose

L.J.-S.Y. Department of Neurosurgery, University of Michigan, 1500 E. Medical Center Dr, Room 3552 TC, Ann Arbor, Ml 48109-5338. Address correspondence to: L.J.-S.Y.; e-mail: ljsyang@med.umich.edu Disclosure: nothing to disclose

Submitted for publication May 21, 2015; accepted October 29, 2015. 